\title{
Sampling and Analysis of Disaster-Impacted Properties: A Data Management Case Study From Ground Zero
}

\author{
S. Badger*, B. Scheetz**, R.J. Lee*, F.S. Schwerer*, K. Rickabaugh*, and C. Bunker* \\ * $\quad$ RJ Lee Group, 350 Hochberg Road, Monroeville, PA 15146 \\ ** Materials Research Institute, The Pennsylvania State University, University Park, PA 16803
}

The collapse of the World Trade Center (WTC) Towers (Figure 1) on September 11, 2001 proceeded subject to crisis-modified environmental standards [1]. Dioxins, PCBs, asbestos, and heavy metal contaminants were reported in air samples collected by the United States Environmental Protection Agency (EPA) and others at least a month after the 9/11 incident. Other efforts to evaluate the environmental impact and potential short and long-term risks posed by toxic substances released by the disaster, and the impact adjacent to WTC properties created even greater challenges for sampling, analysis, and data management. The amount of sampling required is enormous; maintaining data integrity is essential; data needs to be shared among geographically dispersed interested parties (e.g., analytical laboratories, consultants, and experts); and the entire process must be conducted expeditiously.

Assessing the environmental implications resulting from the WTC disaster requires the coordination of field sampling, the use of multiple analytical methods, and integration of personnel from multiple disciplines including microscopy, engineering, toxicology, and environmental risk assessment. Optical light microscopy and electron beam microanalysis techniques are used to characterize the dusts as well as to determine concentrations of particulate contaminants. Microscopy techniques are also utilized to provide forensic information about the source(s) of dusts based on "signature" particle types. The microscopy data must also be compared and contrasted with other relevant analytical data such as bulk organic and inorganic chemistry results.

Organic analyses are used to assess the quantities of dioxins/furans, polychlorinated biphenols (PCBs) and, and polynuclear aromatic hydrocarbons (PNAs). Inorganic methods are used to determine the presence and quantities of metals, including mercury, beryllium and lead as well as other species such as crystalline silica. All of these data are being collated, evaluated and used in assessing the present and future issues related to this disaster.

Sample collection conditions are difficult. Sampling teams are required to wear safety equipment including full-face respirators and other safety garments. Samples are collected for a variety of analytical protocols and need to be identified and associated with an extensive amount of site specific field data.

In order to effectively create and manage the large volumes of data produced by studies in which we are participating, RJ Lee Group implemented an integrated system extending from the field data collection and sampling, through the laboratory processing and to net-based review and reporting systems. Pre-printed sets of chemically resistant bar-code labels and rugged, handheld Personal Data Acquisition (PDA) units maximize the efficiency and integrity of the data collection process. An extensive acquisition program was developed for the PDAs using graphical user interface features such as drop down lists to simplify the demands on the field personnel. The field data were 
transferred electronically back to the central lab as XML files; the bar-coded sample containers and chains of custody information were sent by courier.

PDAs were also configured for use at TEM and SEM instruments to expedite recording and transmittal of analytical results for the large number of samples. The PDA software scanned sample and aliquot bar-codes to link the microscopy specimen to the field data, implemented user- and instrument-interfaces to capture analytical information, and exported the results as XML (data) and TIFF (image) objects. Field data and lab results are collected and subjected to initial quality validation checks at the central lab and then transferred electronically to the central Lotus Notes server.

The data management system is web-centric, using XML files and HTTP to transfer data and to provide multi-user access to results and sharing of findings. To ensure integrity, a single data repository was implemented. Interfaces were created to facilitate web-access to the field and lab results by consultants and analysts.

This paper will provide a high-level review of the data management systems, and a review of the some of the unique aspects of the contaminants and their associations related to the World Trade Center Disaster. Particular attention will be focused on the potential health implications of chrysotile asbestos that was fibrilized by the explosions and fires, and which had various metals and organic compounds deposited on the fibril surfaces.

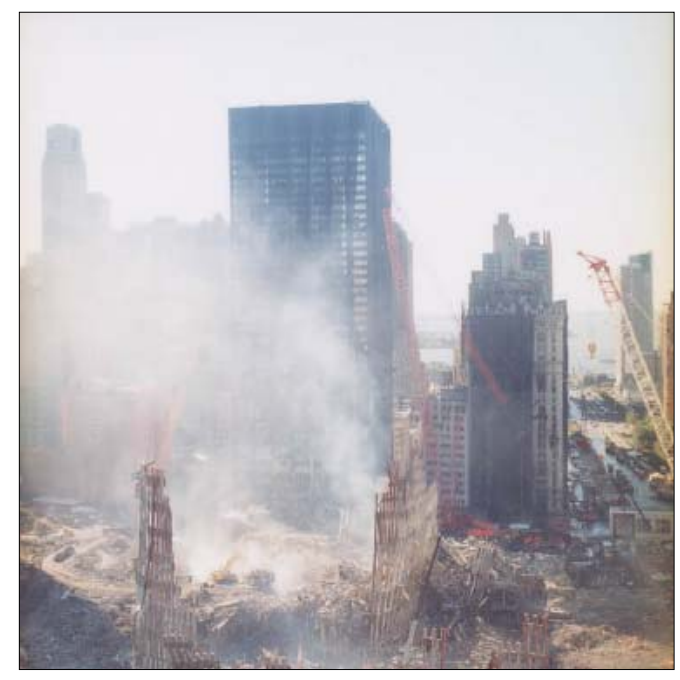

Fig. 1. World Trade Center Site showing damage incurred from the 9/11 attacks and subsequent burning of debris. The photo was taken on January $16^{\text {th }}, 2002$ from the northwest side of the WTC site facing south).

\section{References}

[1] W. Langewiesche, American Ground, North Point Press, New York, 2002. 